Original Research Paper

\title{
Stroke Associated with Infective Endocarditis. Frequency and Prognostic Implications
}

\author{
Juan José Mengual Chirife, Manuel Gómez-Choco and Ester Moral \\ Department of Neurology, Complex Hospitalari Moisès Broggi, Spain
}

\author{
Article history \\ Received: 13-04-2021 \\ Revised: 19-07-2021 \\ Accepted: 31-07-2021 \\ Corresponding Author: \\ Juan José Mengual Chirife \\ Department of Neurology, \\ Complex Hospitalari Moisès \\ Broggi, Spain \\ Email: \\ JuanJose.MengualChirifie@san \\ itatintegral.org
}

\begin{abstract}
Stroke can be one of the most severe complications of Infective Endocarditis (IE) and it happens in $14 \%$ of patients with IE. Therefore, we aimed to review stroke frequency in patients diagnosed with IE in our hospital from 2010 to 2020. We defined it as IE-Associated Stroke (IEAS) when the stroke happened one month before or after IE's diagnosis. Sixty-six patients were diagnosed with IE during this period time and 15 (22\%) suffered IEAS: 13 ischemic strokes, one Subarachnoid Hemorrhage (SAH) and one parenchymal hemorrhage with SAH. Patients with IEAS were more frequently men (80\% vs $51 \% \mathrm{p}=0.042)$ and had less atrial fibrillation $(13.3 \%$ vs $37.3 \%, \mathrm{p}=0.071)$. Although not statistically significant, Streptococcus sp (53\% vs. $44.9 \%)$ and Staphylococcus aureus $(33.3 \%$ vs. $16.3 \%)$ were more frequent in patients with IEAS. Mortality was increased in patients with IEAS (60\% vs $13.7 \%, p=0.001)$ especially in patients where stroke diagnosis was posterior to IE (88.9\% vs $16.7 \%, \mathrm{p}=0.011)$. Hypertension $(93.8 \%$ vs $63 \%, \mathrm{p}=0.013)$ and diabetes $(53.2 \%$ vs $30 \%$, p0 $=0.052$ ) were also more frequent in those patients who died. In multivariate analysis, hypertension (OR 13.7 95\% CI 1.4-138.4) and IEAS (OR 12.8 95\% CI 2.8-58) were independently associated with death. The frequency of IEAS in our patients is comparable to what has been described in the literature. IEAS was associated with increased mortality.
\end{abstract}

Keywords: Infectious Endocarditis, Ischemic Stroke, Hemorrhagic Stroke

\section{Introduction}

It has gone more than a century since Sir William Osler made the first exhaustive description of Infective Endocarditis (IE) (Levy, 1985) in the XIX century. However, despite the advances in diagnosis and treatment, IE remains a clinical challenge nowadays. Moreover, the complexity of IE makes it necessary to set multidisciplinary "endocarditis teams" as has been recommended by the European Cardiology Society in a consensus statement in 2015 (Habib et al., 2015).

In high-income countries an increasingly older population and the associated comorbidities are major determinants for the development of IE. In addition, degenerative valvular disease, prosthetic valves, intracardiac implantable devices and invasive medical procedures also play a role in developing IE. However, in low-middle income countries, rheumatic heart disease and congenital heart diseases still remain the most important factors (Cahill and Prendergast, 2016; Murdoch et al., 2009; Njuguna et al., 2017).

IE is diagnosed with the modified Duke criteria as definite, possible and rejected (Li et al., 2000). Vascular events, such as major arterial emboli, mycotic aneurysms and intracranial hemorrhages, are included in IE's diagnosis's minor criteria. In this setting, stroke can appear as an extracardiac complication of IE in $14 \%$ of the cases (García-Cabrera et al., 2013) and usually carries a poor clinical prognosis (García-Cabrera et al., 2013; Thuny et al., 2007; Morris et al., 2014).

Table 1: Microbiologic etiology in patients with IE

\begin{tabular}{lll}
\hline Microorganism & $\begin{array}{l}\text { Frequency }(\%) \\
\text { Europe }\end{array}$ & North America \\
\hline Streptococcus & 31 & 17 \\
Staphylococcus aureus & 28 & 43 \\
CoNS & 13 & 12 \\
Enterococcus & 9 & 13 \\
Fungal & 1 & 3 \\
HACEK & 2 & 0 \\
Polymicrobial & 1 & 1 \\
Culture negative & 10 & 7 \\
Other & 5 & 4
\end{tabular}

Adapted from Murdoch et al. (2009). CoNS, coagulase-negative staphylococci; HACEK, Haemophilus spp., Aggregatibacter spp., Cardiobacterium hominis, Eikenella corrodens and Kingella spp.; VGS, viridans group streptococci 
Meningitis, encephalopathy, seizures and cerebral abscess are other well-known complications of IE (Cahill and Prendergast, 2016; Hoen and Duval, 2013; Heiro et al., 2000; Bashore et al., 2006; Jones and Siekert, 1989; Greenlee and Mandell, 1973). Accordingly, neurology specialists may be involved and play an essential role in managing patients with IE.

The causal agents of IE are varied, in Table 1 we mentioned the most frequently involved.

Our study aimed to describe stroke frequency during the last ten years in patients diagnosed with IE in our hospital and describe the main clinical characteristics and prognostic implications.

\section{Methods}

Moisès Broggi Hospital is located in Barcelona's south metropolitan area and attends a population of 422.000 inhabitants. We selected from our local electronic records patients that had been diagnosed with IE (ICD-10 code I33) and ischemic stroke (ICD-10 codes I63), hemorrhagic stroke (ICD-10 codes I61), or subarachnoid hemorrhage (SAH, ICD-10 codes I60). Finally, we defined IE-associated stroke (IEAS) when stroke happened one month before or after IE diagnosis. We collected the following variables: age, sex, hypertension, diabetes, dyslipidemia, Chronic Kidney Disease (CKD), Ischemic Heart Disease (IHD), Atrial Fibrillation (AF), presence of Implantable Cardiac Devices (ICD), affected valve, microorganism causing IE, valve replacement surgery, the temporal relationship between stroke and IE diagnosis and death. Patients diagnosed with IE were divided into IEAS (IEAS+) patients and those without IEAS (IEAS).
Statistical analysis was performed with IBM SPSS 26. Firstly, we compared patients with and without IEAS. Secondly, we selected those patients with IEAS and compared those in which stroke diagnosis preceded the diagnosis of IE and those with a stroke posterior to IE diagnosis. Continuous variables were described as means and Standard Deviations (SD) and categorical variables were expressed as percentages. Frequency tables were analyzed using Pearson's chi-squared test or Fisher's test, as appropriate. Quantitative differences between groups were compared using Student's t-test, ANOVA, or Mann-Whitney U test, as appropriate. We considered as statistically significant $\mathrm{p}$ values of $<0.05$ and all hypotheses were two-sided. When required, we used multivariate analysis to adjust for confounding variables.

\section{Results}

Sixty-six patients were diagnosed with IE in our hospital from 2010 to 2020 . The main demographic and clinical characteristics are described in Table 2. Fifteen patients $(22.7 \%)$ suffered an IEAS: 13 ischemic strokes, one Subarachnoid Hemorrhage (SAH) and one parenchymal bleeding with SAH. In addition, the patient with an isolated SAH underwent a brain angiogram and was diagnosed with a mycotic aneurysm (Fig. 1).

The middle cerebral artery was the arterial territory most frequently involved in patients with ischemic stroke and 10 out of $13(77 \%)$ patients with ischemic stroke had acute ischemic lesions in more than one arterial territory, suggesting the embolic nature.

Stroke preceded the diagnosis of IE in 6 patients (42\%). Detailed characteristics of patients with IEAS appear in Table 3.

Table 2: Basal characteristics of the 66 patients diagnosed with IE

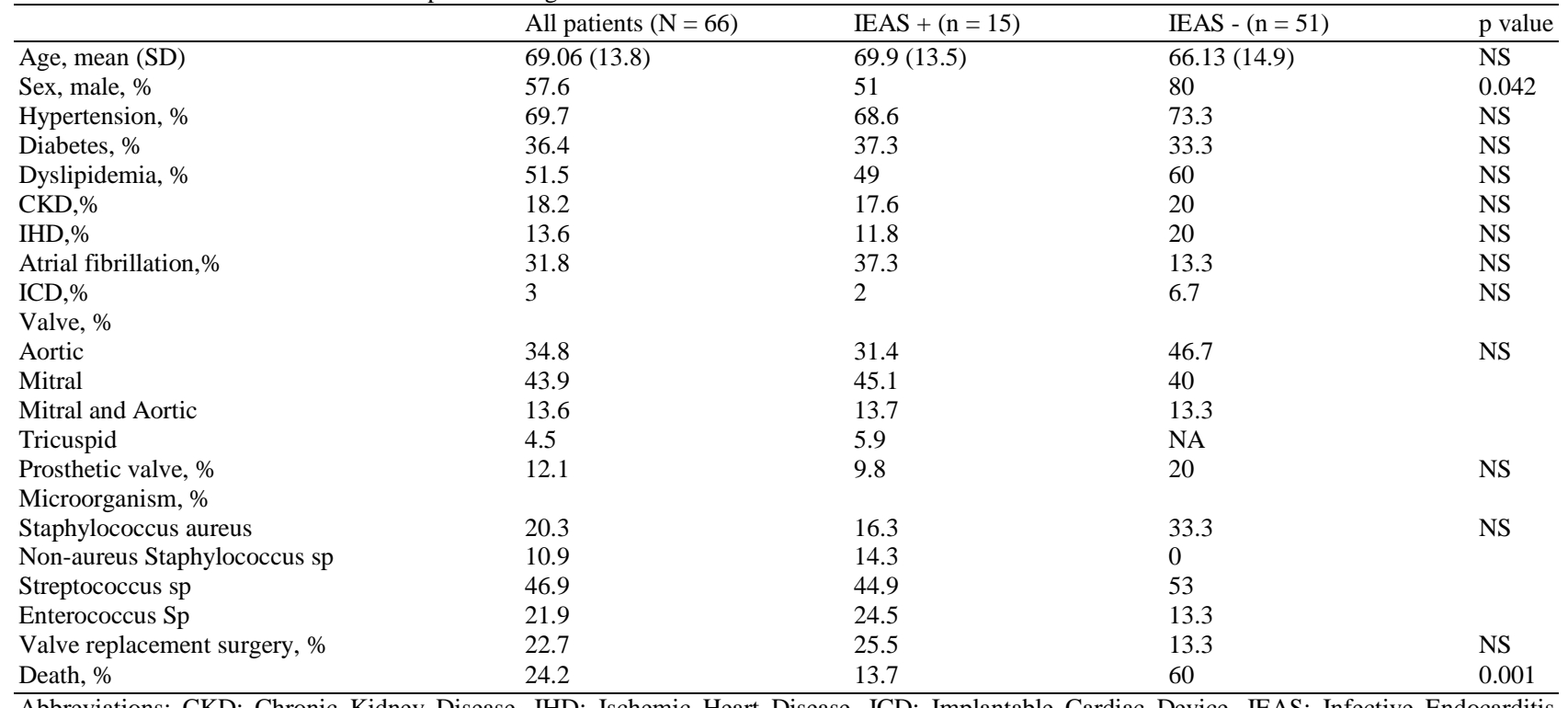

Abbreviations: CKD: Chronic Kidney Disease, IHD: Ischemic Heart Disease, ICD: Implantable Cardiac Device, IEAS: Infective Endocarditis Associated Stroke, NA: not applicable, NS: not significant 


\begin{tabular}{|c|c|c|c|c|c|c|c|c|c|}
\hline Patient $\mathrm{N}^{\mathrm{o}}$ & Age & Sex & $\begin{array}{l}\text { Afected } \\
\text { Valve }\end{array}$ & Microorganism & $\begin{array}{l}\text { Stroke } \\
\text { subtype }\end{array}$ & Location & $\begin{array}{l}\text { IE debut } \\
\text { with stroke }\end{array}$ & $\begin{array}{l}\text { Time from } \\
\text { IE to stroke }\end{array}$ & Death \\
\hline 1 & 89 & $\mathrm{~F}$ & Aortic-Mitral & S. Viridans & IS & L-MCA & Yes & NA & No \\
\hline 2 & 80 & $\mathrm{~F}$ & Mitral & S. Bovis & IS & L and R-MCA, L and R-PCA & No & 15 days & Yes \\
\hline 3 & 80 & $\mathrm{~F}$ & Mitral & S. Viridans & IS & L-MCA, L-PCA & No & 1 month & Yes \\
\hline 4 & 86 & M & Aortic & S. Salivarius & IS & $\mathrm{L}$ and $\mathrm{R}-\mathrm{MCA}$, brainstem & No & 21 days & Yes \\
\hline 5 & 71 & M & Prosthetic Mechanical Aortic & E. Faecalis & IS & SCA & Yes & NA & No \\
\hline 6 & 66 & M & Prosthetic Biological mitral & MRSA & IS & L and R-MCA;brainstem & Yes & NA & Yes \\
\hline 8 & 62 & M & Aortic & S. Oralis & IS & Brainstem & Yes & NA & No \\
\hline 9 & 51 & M & Aortic-Mitral & E. Faecalis & SAH & Mycotic aneurism-L-MCA & No & 8 days & Yes \\
\hline 10 & 63 & M & Mitral & S. Aureus & IS & $\mathrm{L}$ and $\mathrm{R}-\mathrm{MCA}$ & No & 10 days & Yes \\
\hline 11 & 47 & M & Aortic & S. Aureus & IS & L and R-MCA, L and R-PCA & No & 3 days & Yes \\
\hline 12 & 49 & M & Aortic & S. Aureus & IS & L and R-MCA, L and R-PCA & Yes & $\mathrm{NA}$ & No \\
\hline 13 & 80 & M & Aortic & S. Epidermidis & IS & L and R-MCA; R-PICA & No & 5 days & Yes \\
\hline 14 & 47 & M & Mitral & S. Aureus & IS & $\mathrm{L}$ and $\mathrm{R}-\mathrm{MCA}$ & Yes & NA & No \\
\hline 15 & 49 & M & Mitral & S. Oralis & IS & L and R-MCA; R-PICA & No & 3 weeks & No \\
\hline
\end{tabular}

Abbreviations: IS: Ischemic stroke; HS; hemorrhagic stroke; L: left, R: Right, MCA: Middle cerebral artery; PCA: Posterior cerebral artery; SCA: Superior cerebellar artery; SAH: Subarachnoid hemorrhage; PICA: Postero inferior cerebelar artery, NA: Not applicable.
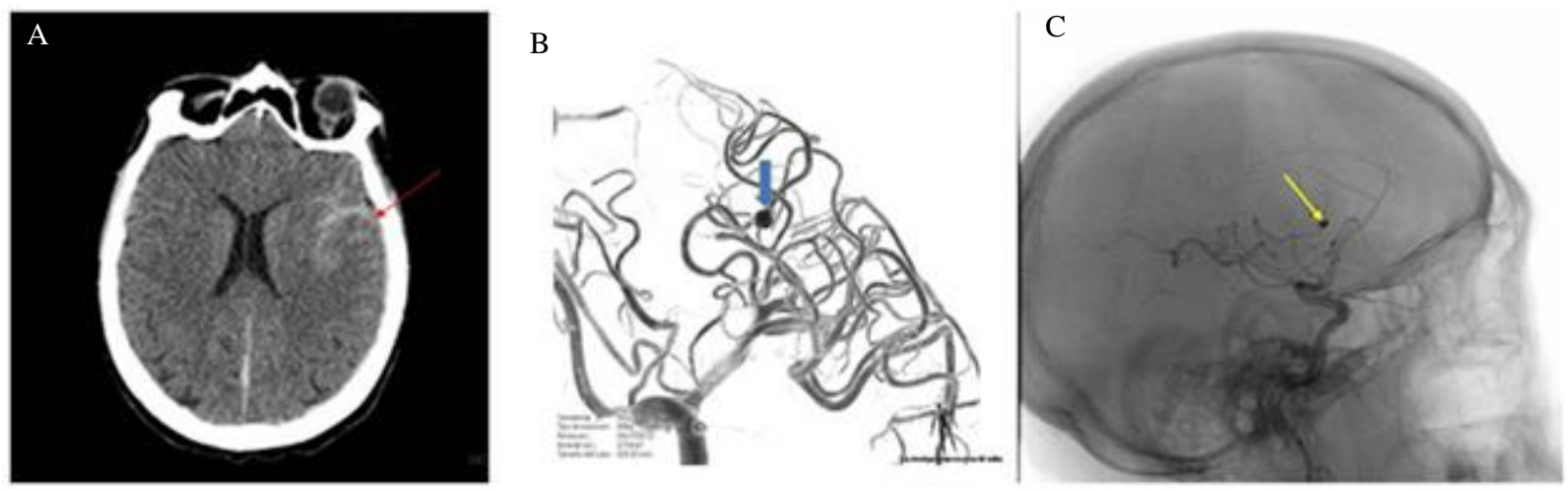

Fig. 1: A 51 years old male with Mitro-Aortic IE complicated with hemorrhagic stroke due to a mycotic aneurism. A. Basal CT showing subarachnoid hemorrhage (red arrow). B. Mycotic aneurysm in a distal ramification of the MCA (green arrow). $\mathrm{C}$ : Coil implanted in the mycotic aneurysm (yellow arrow)

Patients with IEAS were more frequently men $(80 \%$ vs $51 \% \mathrm{p}=0.042)$ and had less atrial fibrillation $(13.3 \%$ vs $37.3 \%, p=0.071)$. Streptococcus sp. (53\% vs. $44.9 \%)$ and Staphylococcus aureus $(33.3 \%$ vs. $16.3 \%)$ were more frequent in patients with IEAS, although the difference was not statistically significant. Mortality was markedly increased in patients with IEAS $(60 \%$ vs. $13.7 \%, \mathrm{p}=0.001)$, especially in those with stroke posterior to IE diagnosis $(88.9 \%$ vs. $16.7 \% \mathrm{p}=0.011)$. Hypertension $(93.8 \%$ vs $63 \%, \mathrm{p}=0.013)$ and diabetes $(53.2 \%$ vs $30 \%, \mathrm{p} 0=0.052)$ were also more frequent in those patients who died. In multivariate analysis, hypertension (OR 13.7 95\% CI 1.4-138.4) and IEAS (OR 12.8 95\%CI 2.8-58) were independently associated with death.

\section{Discussion}

The incidence of IE is 3-9 cases per 100,000 per year (Cao et al., 2018; Hoen and Duval, 2013) and it has been stable over last decades, despite the advance of preventive medicine strategies and better diagnostics tools (Hoen and Duval, 2013; Murdoch et al., 2009). Stroke in the IE setting is infrequent (percentages can vary from 10-50\% for embolic events, $65 \%$ affecting the central nervous system) (Heiro et al., 2000; Morris et al., 2014) but carries an ominous prognosis. For example, in our series of patients with IE for ten years, stroke appears as a complication of IE in $22.7 \%$. This percentage is similar to previously reported (Thuny et al., 2007; Sotero et al., 2019; Anderson et al., 2003; Fabri Jr et al., 2006; Barsic et al., 2013; Hasbun et al., 2003), but it might be even higher since the risk of stroke remains increased in the first year after the IE diagnosis (Merkler et al., 2015; Østergaard et al., 2019). The observed mortality in our series is also comparable to other studies (García-Cabrera et al., 2013; Anderson et al., 2003; Hasbun et al., 2003; Hobohm et al., 2016).

However, Dickerman et al. (2007) showed us that the stroke rate during the first week of antibiotic therapy was 4.82 of 1000 patient-days. Still, this rate fell by $65 \%$ in the second week of treatment to 1.71 of 1000 patient days $(p<0.001)$ and continued constantly falling after the start of antibiotic therapy. This could be explained by the breakdown of the vegetation formed by inflammatory cells, endothelial cells, invading microorganisms, platelets and red blood cells 
with antimicrobial therapy, leading to a decrease in the vegetation's size and or stabilization.

We defined IEAS when the stroke happened either one month before or after the IE diagnosis because the risk is maximal during this period and more likely a causative relationship with IE. Compared to other studies, we found a similar pattern of microorganisms and valvular involvement (Hoen and Duval, 2013; Bashore et al., 2006; Hobohm et al., 2016; Lee et al., 2014), being S. aureus the most common single microorganism responsible for IE. This phenomenon is related to the increased healthcare contact in the general population and the chronification of diseases (Cahill and Prendergast, 2016; Bashore et al., 2006; Jones Jr and Siekert, 1989; Greenlee and Mandell, 1973; Murdoch et al., 2009), but possibly Streptococcus sp. still represents the foremost responsible for IE in the low and middle-income countries with a progressive increment of S. aureus infection (Cahill and Prendergast, 2016; Njuguna et al., 2017). Similarly, we also found the classical multi-territorial involvement described for ischemic stroke caused by IE (Jones Jr and Siekert, 1989; Greenlee and Mandell, 1973; Lee et al., 2014; Singhal et al., 2002), being the middle cerebral artery the most affected territory. In fact, 3 of our patients with ischemic stroke before IE diagnosis had a single territorial infarct. Lee et al. (2014; Singhal et al., 2002) described four patterns of ischemic lesions in DWI MRI (A) single lesion, (B) territorial infarction, (C) disseminated punctate lesions and (D) numerous small $(<10 \mathrm{~mm})$ and medium $(10$ to $30 \mathrm{~mm})$ or large $(>30 \mathrm{~mm})$ lesions in multiple territories. It's crucial to highlight that only pattern D was exhibited in Non-Bacterial Thrombotic Endocarditis (NBTE) patients. Therefore, although brain imaging can show different patterns suggestive of an underlying IE, other clinical features such as fever, raised acute phase reactants or the presence of a new cardiac murmur must be taken into account.

The main result of our study is that stroke is associated with significantly increased mortality in patients with IE, especially when IE diagnosis preceded the stroke. Similar findings were reported by Epaulard et al. (2009). In a small study with 32 patients with IE, mortality was higher in patients with stroke after IE diagnosis (Murdoch et al., 2009). Patients with acute stroke had probably been admitted to stroke units, with early neuroimaging and cardiac workout that had allowed an earlier detection and treatment of IE. However, the difference in mortality between patients diagnosed with stroke before or after IE should be confirmed with a larger sample. This association of IEAS with mortality was independent of hypertension, another factor associated with increased mortality and probably the fatal evolution might be caused by stroke occurrence.

This study has significant limitations; apart from the limited number of patients and its retrospective nature, we cannot assure that the patients had been treated with coordinated protocols. This might impact mortality, for instance, the lack of cardiac surgery service inwards. However, we think that the important difference in mortality between both groups makes this point unlikely. Another caveat is that only patients with focal neurological signs were evaluated by a neurologist and went under a selection bias neuroimaging. It's well known that silent ischemic lesions are detected with routine neuroimaging, preferably cerebral MRI, but the ideal time and clinical relevance are still unknown and need further evaluation.

In conclusion, Stroke is an infrequent but serious complication of IE, associated with a high mortality, especially when stroke happens after IE diagnosis. The causing microorganisms are similar to previous series. A high grade of clinical suspicion and early diagnosis of IE might help to improve clinical prognosis in these patients.

\section{Acknowledgement}

We thank Jordi Vericat for his technical assisstance.

\section{Author's Contributions}

Juan José Mengual Chirife: Collected the data and drafted the manuscript.

Manuel Gómez-Choco: Carried out the statistical analysis and drafted the manuscript.

Ester Moral: Designed the study and made a critical review of the manuscript.

\section{Ethics}

The investigation conforms to the principles outlined in the declaration of Helsinki. The need for written informed consent was waived for the retrospective character of the study.

\section{References}

Anderson, D. J., Goldstein, L. B., Wilkinson, W. E., Corey, G. R., Cabell, C. H., Sanders, L. L., \& Sexton, D. J. (2003). Stroke location, characterization, severity and outcome in mitral vs aortic valve endocarditis. Neurology, 61(10), 1341-1346. https://doi.org/10.1212/01.WNL.0000094359.479 29.E4

Barsic, B., Dickerman, S., Krajinovic, V., Pappas, P., Altclas, J., Carosi, G., ... \& International Collaboration on Endocarditis-Prospective Cohort Study (ICE-PCS) Investigators. (2013). Influence of the timing of cardiac surgery on the outcome of patients with infective endocarditis and stroke. Clinical Infectious Diseases, 56(2), 209-217. https://doi.org/10.1093/cid/cis878

Bashore, T. M., Cabell, C., \& Fowler Jr, V. (2006). Update on infective endocarditis. Current problems in Cardiology, 31(4), 274-352.

https://doi.org/10.1016/j.cpcardiol.2005.12.001 
Cahill, T. J., \& Prendergast, B. D. (2016). Infective endocarditis. Lancet. 27, 387(10021), 882-93. doi.org/10.1016/S0140-6736(15)00067-7

Cao, G. F., Liu, W., \& Bi, Q. (2018). Stroke in patients with infective endocarditis: A 15-year single-center cohort study. European Neurology, 80(3-4), 171-178. doi.org/10.1159/000495149

Dickerman, S. A., Abrutyn, E., Barsic, B., Bouza, E., Cecchi, E., Moreno, A., ... \& Cabell, C. H. (2007). The relationship between the initiation of antimicrobial therapy and the incidence of stroke in infective endocarditis: An analysis from the ICE Prospective Cohort Study (ICE-PCS). American Heart Journal, 154(6), 1086-1094. doi.org/10.1016/j.ahj.2007.07.023

Epaulard, O., Roch, N., Potton, L., Pavese, P., Brion, J. P., \& Stahl, J. P. (2009). Infective endocarditis-related stroke: diagnostic delay and prognostic factors. Scandinavian Journal of Infectious Diseases, 41(8), 558-562. doi.org/10.1080/00365540902984701

Fabri Jr, J., Issa, V. S., Pomerantzeff, P. M., Grinberg, M., Barretto, A. C. P., \& Mansur, A. J. (2006). Time-related distribution, risk factors and prognostic influence of embolism in patients with left-sided infective endocarditis. International Journal of Cardiology, 110(3), 334-339. doi.org/10.1016/j.ijcard.2005.07.016

García-Cabrera, E., Fernández-Hidalgo, N., Almirante, B., Ivanova-Georgieva, R., Noureddine, M., Plata, A., ... \& González, A. D. A. (2013). Neurological complications of infective endocarditis: risk factors, outcome and impact of cardiac surgery: a multicenter observational study. Circulation, 127(23), 2272-2284. doi.org/10.1161/CIRCULATIONAHA.112.000813

Greenlee, J. E., \& Mandell, G. L. (1973). Neurological manifestations of infective endocarditis: a review. Stroke, 4(6), 958-963.

doi.org/10.1161/01.STR.4.6.958

Habib, G., Lancellotti, P., Antunes, M. J., Bongiorni, M. G., Casalta, J. P., Del Zotti, F., ... \& Zamorano, J. L. (2015). 2015 ESC guidelines for the management of infective endocarditis: the task force for the management of infective endocarditis of the European Society of Cardiology (ESC) endorsed by: European Association for Cardio-Thoracic Surgery (EACTS), the European Association of Nuclear Medicine (EANM). European Heart Journal, 36(44), 3075-3128. doi.org/10.1093/eurheartj/ehv319

Hasbun, R., Vikram, H. R., Barakat, L. A., Buenconsejo, J., \& Quagliarello, V. J. (2003). Complicated left-sided native valve endocarditis in adults: risk classification for mortality. JAMA, 289(15), 1933-1940.

doi.org/10.1001/jama.289.15.1933
Heiro, M., Nikoskelainen, J., Engblom, E., Kotilainen, E., Marttila, R., \& Kotilainen, P. (2000). Neurologic manifestations of infective endocarditis: A 17-year experience in a teaching hospital in Finland. Archives of Internal Medicine, 160(18), 2781-2787. doi.org/10.1001/archinte.160.18.2781

Hobohm, C., Hagendorff, A., Schulz, S., Fritzsch, D., Budig, S., Stöbe, S., \& Michalski, D. (2016). Clinical presentation and multi-parametric screening surrogates of ischemic stroke patients suffering from infective endocarditis. Cerebrovascular Diseases, 41(1-2), 60-67. doi.org/10.1159/000442005

Hoen, B., \& Duval, X. (2013). The clinical problem. N Engl J Med, 368, 1425-33. doi.org/10.1056/NEJMcp1206782

Jones Jr, H. R., \& Siekert, R. G. (1989). Neurological manifestations of infective endocarditis. Review of clinical and therapeutic challenges. Brain: A Journal of Neurology, 112, 1295-1315. doi.org/10.1093/brain/112.5.1295

Lee, S. J., Oh, S. S., Lim, D. S., Na, C. Y., \& Kim, J. H. (2014). Clinical significance of cerebrovascular complications in patients with acute infective endocarditis: a retrospective analysis of a 12-year single-center experience. BMC Neurology, 14(1), 1-9. doi.org/10.1186/1471-2377-14-30

Levy, D. M. (1985). Centenary of William Osler's 1885 Gulstonian lectures and their place in the history of bacterial endocarditis. Journal of the Royal Society of Medicine, 78(12), 1039-1046. doi.org/10.1177/014107688507801213

Li, J. S., Sexton, D. J., Mick, N., Nettles, R., Fowler Jr, V. G., Ryan, T., ... \& Corey, G. R. (2000). Proposed modifications to the Duke criteria for the diagnosis of infective endocarditis. Clinical Infectious Diseases, 30(4), 633-638. doi.org/10.1086/313753

Merkler, A. E., Chu, S. Y., Lerario, M. P., Navi, B. B., \& Kamel, H. (2015). Temporal relationship between infective endocarditis and stroke. Neurology, 85(6), 512-516. doi.org/10.1212/WNL.0000000000001835

Morris, N. A., Matiello, M., Lyons, J. L., \& Samuels, M. A. (2014). Neurologic complications in infective endocarditis: identification, management and impact on cardiac surgery. The Neurohospitalist, 4(4), 213-222. doi.org/10.1177/1941874414537077

Murdoch, D. R., Corey, G. R., Hoen, B., Miró, J. M., Fowler, V. G., Bayer, A. S., ... \& International Collaboration on Endocarditis-Prospective Cohort Study (ICE-PCS) Investigators. (2009). Clinical presentation, etiology and outcome of infective endocarditis in the 21st century: The International Collaboration on Endocarditis-Prospective Cohort Study. Archives of Internal Medicine, 169(5), 463-473. doi.org/10.1001/archinternmed.2008.603 
Njuguna, B., Gardner, A., Karwa, R., \& Delahaye, F. (2017). Infective endocarditis in low-and middle-income countries. Cardiology Clinics, 35(1), 153-163. doi.org/10.1016/j.ccl.2016.08.011

Østergaard, L. andersson, N. W., Kristensen, S. L., Dahl, A., Bundgaard, H., Iversen, K., ... \& Fosbøl, E. L. (2019). Risk of stroke subsequent to infective endocarditis: a nationwide study. American Heart Journal, 212, 144-151. doi.org/10.1016/j.ahj.2019.03.010

Singhal, A. B., Topcuoglu, M. A., \& Buonanno, F. S. (2002). Acute ischemic stroke patterns in infective and nonbacterial thrombotic endocarditis: A diffusionweighted magnetic resonance imaging study. Stroke, 33(5), 1267-1273.

doi.org/10.1161/01.STR.0000015029.91577.36
Sotero, F. D., Rosário, M., Fonseca, A. C., \& Ferro, J. M. (2019). Neurological complications of infective endocarditis. Current Neurology and Neuroscience Reports, 19(5), 1-8. doi.org/10.1007/s11910-0190935-x

Thuny, F., Avierinos, J. F., Tribouilloy, C., Giorgi, R., Casalta, J. P., Milandre, L., ... \& Habib, G. (2007). Impact of cerebrovascular complications on mortality and neurologic outcome during infective endocarditis: A prospective multicentre study. European Heart Journal, 28(9), 1155-1161. doi.org/10.1093/eurheartj/ehm005 